Rochester Institute of Technology

RIT Scholar Works

10-3-2018

\title{
Superhydrophobic Waveguide: Liquid-core air-cladding waveguide platform for optofluidics
}

\author{
Ke Du \\ Rochester Institute of Technology \\ Ishan Wathuthanthri \\ Northrop Grumman Mission Systems \\ Junjun Ding \\ Alfred University \\ Chang-Hwan Choi \\ Stephens Institute of Technology
}

Follow this and additional works at: https://scholarworks.rit.edu/article

\section{Recommended Citation}

K. Du, I. Wathuthanthri, J. Ding, C.H. Choi, "Superhydrophobic waveguide: Liquid-core air-cladding waveguide platform for optofluidics," Appl. Phys. Lett. 113, 143701 (2018); https://doi.org/10.1063/ 1.5049692

This Article is brought to you for free and open access by the Faculty \& Staff Scholarship at RIT Scholar Works. It has been accepted for inclusion in Articles by an authorized administrator of RIT Scholar Works. For more information, please contact ritscholarworks@rit.edu. 


\title{
Superhydrophobic waveguide: Liquid-core air-cladding waveguide platform for optofluidics
}

\author{
Ke Du, ${ }^{1,2}$ Ishan Wathuthanthri, ${ }^{1,3}$ Junjun Ding, ${ }^{1,4}$ and Chang-Hwan Choi ${ }^{1, a)}$ \\ ${ }^{1}$ Department of Mechanical Engineering, Stevens Institute of Technology, Hoboken, New Jersey 07030, USA \\ ${ }^{2}$ Department of Mechanical Engineering, Rochester Institute of Technology, Rochester, New York 14623, USA \\ ${ }^{3}$ Northrop Grumman Mission Systems, Advanced Technology Labs, Linthicum, Maryland 21090, USA \\ ${ }^{4}$ New York State College of Ceramics, Alfred University, Alfred, New York 14802, USA
}

(Received 24 July 2018; accepted 18 September 2018; published online 3 October 2018)

\begin{abstract}
In this paper, we present an optofluidic waveguide platform consisting of liquid as a core material and air as cladding, enabled by using a superhydrophobic channel featured with hydrophobized high-aspect-ratio sharp-tip nanostructures. The contact of the liquid core with the superhydrophobic channel wall is minimized with an air layer retained between them so that the effective refractive index of the cladding layer is close to that of air. Thus, when light is introduced through the core liquid having a higher refractive index than that of the cladding air at the incident angle parallel to the channel direction less than a critical angle, it is reflected at the liquid-gas interface by the total internal reflection. When the cladding layer is filled with water (i.e., Wenzel state), the waveguide losses for the incident angles of 0 and $10^{\circ}$ were $\sim 3.9$ and $\sim 6.8 \mathrm{~dB} / \mathrm{cm}$, respectively. In contrast, when the cladding layer is retained with air (i.e., Cassie-Baxter state), the waveguide losses for the same incident angles were as low as $\sim 0.1$ and $\sim 1.8 \mathrm{~dB} / \mathrm{cm}$, respectively. The significantly lowered waveguide losses at the Cassie-Baxter state indicate that superhydrophobic channels can provide the effective waveguide platform for optofluidics, exploiting the air layer as the cladding material. Published by AIP Publishing. https://doi.org/10.1063/1.5049692
\end{abstract}

In recent years, optofluidics has drawn great attention, combining the advantages of optics and microfluidics in a single integrated device. ${ }^{1-4}$ Typically, liquid-core waveguide platforms have been developed for biomedical sensing and diagnosing, ${ }^{5-7}$ which requires the light and fluid to share the same path to take advantages of the optofluidic interactions between them. ${ }^{8}$ In the liquid-core waveguide platforms, the refractive index $(n)$ of the cladding material has to be smaller than that of the core liquid such as water $\left(n_{\text {water }}=1.33\right)$ to enable the total internal reflection for the configuration of an index-guided mode. ${ }^{9}$ The index-guided mode can be realized in several different ways. For example, Cho et al. demonstrated the liquid-core waveguide platform using a Teflon AF (amorphous fluoropolymer) layer as the cladding material, ${ }^{10}$ where a Teflon AF layer as thick as $5 \mu \mathrm{m}$ was required to cover the microchannel walls to confine light in the liquid core effectively. Since Teflon AF has a refractive index of 1.31, which is not much different from that of water, the index-guided mode could be achieved only within a relatively small incident angle $\left(<10^{\circ}\right)$. Han et al. demonstrated the liquid-core waveguide platform using hollow-core photonic crystal fiber (PCF) as the cladding material, where the hollow core was filled with water so that the light was guided through index-guided liquid-core PCF rather than bandgapguided hollow-core PCF, which further allowed the surfaceenhanced Raman scattering (SERS) sensing at the interface between liquid and PCF walls. ${ }^{11}$ However, relatively high pumping power for the liquid flow and the associated Joule heating due to the high hydrodynamic friction at the channel walls remain as issues, which could change the temperature

a)Email: cchoi@stevens.edu and properties of the analytes in the liquid core. ${ }^{12,13}$ An antiresonant reflecting optical waveguide mode has also been demonstrated recently based on an anti-resonant Fabry-Pérot reflector, ${ }^{14,15}$ where light can propagate within an oxide layer with a larger refractive index sandwiched by a liquid core and the other oxide layer with a lower refractive index. It can also be integrated with a solid core so that it does not require the liquid core and light to share the same path. However, the fabrication process is complicated and a small channel size is required to reduce propagation loss. ${ }^{16}$ Wolfe et al. demonstrated a liquid-core liquid-cladding waveguide platform, ${ }^{17,18}$ where the interface between the core and cladding could be reconfigured due to the low Reynolds number flow characteristics and adapted continuously by regulating the flow speeds. However, it is not effective to maintain the liquid-liquid interface over a relatively long flow path and operation time. Moreover, most liquids have similar refractive indexes to that of water, which limits the wide application of this mode.

The various challenges and issues in the current indexguided modes demonstrated can be resolved if gas (i.e., air) can be used as the cladding material. Air has a much lower refractive index $\left(n_{\text {air }}=1.0\right)$ than most solid and liquid materials, so it can allow a wider range of incident angles. Air also has much lower viscosity than that of liquid, so it can significantly reduce the hydrodynamic friction and Joule heating at the interface between the core and cladding. On recent advances in microfluidics, superhydrophobic channels have shown that the air layer can be configured and retained on the channel walls using hydrophobic structures on small (i.e., micro and nano) scales which can levitate water due to surface tension effects. ${ }^{19,20}$ However, such superhydrophobic channels 
have not yet been demonstrated as waveguide platforms for the index-guided mode for the liquid-core air-cladding configuration. In this paper, we examine the waveguide coupling efficiency of the superhydrophobic channels that can uniquely allow the index-guided mode on the basis of the liquid-core air-cladding architecture to potentially address and overcome such challenges and issues.

Figure 1 shows the overall scheme of the proposed superhydrophobic waveguiding. The hydrophobic high-aspect-ratio sharp-tip nanostructures levitate water in the core due to roughness-induced superhydrophobicity ${ }^{21}$ and retain air between the liquid core and channel walls. If light is introduced through the core liquid having a higher refractive index than that of the composite cladding layer composed of the solid nanostructures and air with the incident angle less than a critical angle, the light is mostly reflected at the liquid-gas interfaces by the total internal reflection and guided within the liquid core along the channel direction. The effective refractive index $\left(n_{\text {eff }}\right)$ of the composite cladding layer can be estimated by the volume averaging theory ${ }^{22}$ as

$$
n_{\text {eff }}=\sqrt{(1-\phi) n_{\text {air }}^{2}+\phi n_{\text {solid }}^{2}},
$$

where $\phi$ is the solid fraction of the superhydrophobic surface and $n_{\text {solid }}$ is the refractive index of the solid material. With the significantly reduced contact of the solid surface with the liquid core (i.e., small value of $\phi$ ) by the high-aspect-ratio and sharp-tip geometry of the nanostructures, the effective refractive index of the solid-air composite cladding layer can be close to that of air (i.e., $n_{\text {eff }} \cong 1$ ). The high-aspect-ratio sharp-tip geometry of the nanostructures (i.e., small value of $\phi$ ) is also advantageous for reducing the scattering loss of light ${ }^{23}$ and the hydrodynamic friction at the channel walls. ${ }^{24}$

Figure 2(a) shows the scanning electron microscopy (SEM) image of the high-aspect-ratio sharp-tip nanostructures of silicon fabricated by the simple black silicon method $^{25}$ based on a maskless reactive ion etching process (Plasmalab 100, Oxford Instruments) using $\mathrm{O}_{2}$ and $\mathrm{SF}_{6}$ as etching gases. The height of the needle-like nanostructures is $\sim 10 \mu \mathrm{m}$, and the average pitch (distance between adjacent structures) is $\sim 1 \mu \mathrm{m}$, giving the aspect ratio greater than 10 . The radius of the curvature of the tip apex was $\sim 20 \mathrm{~nm}$.

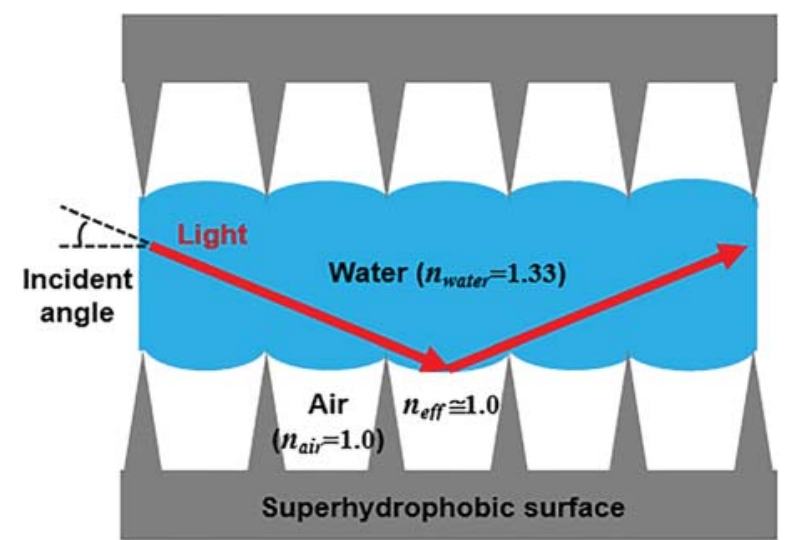

FIG. 1. Schematic of the superhydrophobic waveguide platform. The incident light is mostly reflected at the water/air interface with an effective refractive index close to that of air.

(a)

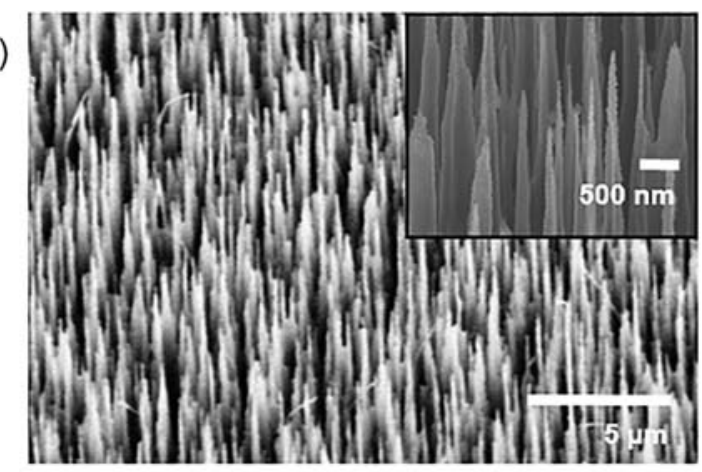

(b) Before PTFE coating (Wenzel state)

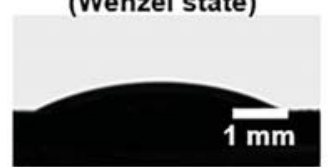

(d)

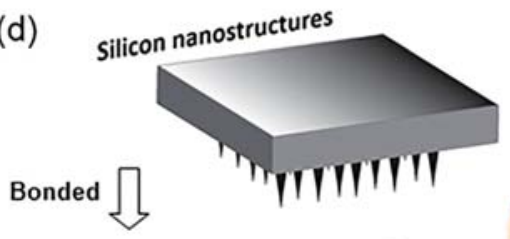

(c) After PTFE coating

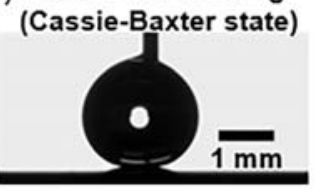


A superhydrophobic channel was prepared using the two silicon substrates featured with the hydrophobized highaspect-ratio sharp-tip nanostructures as the channel walls on top and bottom, as shown in Fig. 2(d). Epoxy was used to bond the silicon substrates to each side of the polydimethylsiloxane (PDMS) layer (1.5 mm thick) whose middle part ( $2.5 \mathrm{~cm}$ long and $1.0 \mathrm{~cm}$ wide) was pre-molded and punched to have open space to serve as a rectangular channel and hold water. At the two ends of the PDMS slab, the inlet and outlet $(1 \mathrm{~mm}$ in diameter) for the introduction of water into the channel were made, using a biopsy punch. Deionized water was then pumped into the channel using a syringe pump. During the measurement of the waveguide coupling efficiency of the superhydrophobic channel, the inlet and outlet were sealed with scotch tapes to minimize any leak of water out of the channel.

The waveguide coupling efficiency of the superhydrophobic channel was examined using the linearly-polarized ultraviolet (UV) light of the $\mathrm{HeCd}$ laser with a wavelength of $325 \mathrm{~nm}$ as the light source, as also illustrated in Fig. 2(d). The beam was spatially filtered through a pinhole before it was introduced to the PDMS channel. The beam was introduced to one end of the other sides than the sides used for the inlet/outlet for water. The incident and transmitted beam powers were measured right before and after the superhydrophobic channel using a UV power detector (Newport Corporation) with resolution of nanowatts and a detection area of $1 \mathrm{~cm}$ by $1 \mathrm{~cm}$. The initial beam power and the beam diameter measured right before the superhydrophobic channel were $480 \mu \mathrm{W}$ and $\sim 500 \mu \mathrm{m}$, respectively. The small beam size $(\sim 500 \mu \mathrm{m})$ relative to that of the liquid core $(1.5 \mathrm{~mm})$ helps to reduce the coupling loss of light at the introduction through the PDMS wall. The large wavelength $(325 \mathrm{~nm})$ relative to that of nanostructures' tips (not more than a couple of tens of nanometers) also helps to reduce the scattering loss of light at the solid tips. ${ }^{23}$ The superhydrophobic channel was mounted on a rotational stage (Thorlabs, Inc.), and the transmitted power through the superhydrophobic waveguide was measured at various incident angles of the beam. Then, the waveguide loss (WL) was estimated using the following equation: ${ }^{30}$

$$
W L=10 \log _{10}\left(\frac{Q_{1}}{Q_{2}}\right),
$$

where $Q_{1}$ and $Q_{2}$ are the laser powers measured at the front and end of the PDMS channel, respectively. The measurement was done for three times to obtain the average and standard deviation values. The waveguide loss of the nanostructured silicon substrates without the PTFE coating (i.e., hydrophilic surfaces with a Wenzel state for the liquid core) was also examined for the channel walls for the comparison to that of the superhydrophobic channels.

Figure 3(a) shows the waveguide loss (WL) for the unit length of the channel. Before measuring the waveguide losses for the hydrophilic (Wenzel state) and superhydrophobic (Cassie-Baxter state) channels, those for the channels without water filled were also measured to estimate the losses through the PDMS walls and air at the various incident angles of the beam. As observed in Fig. 3(a), without water filled, the waveguide losses for hydrophilic and
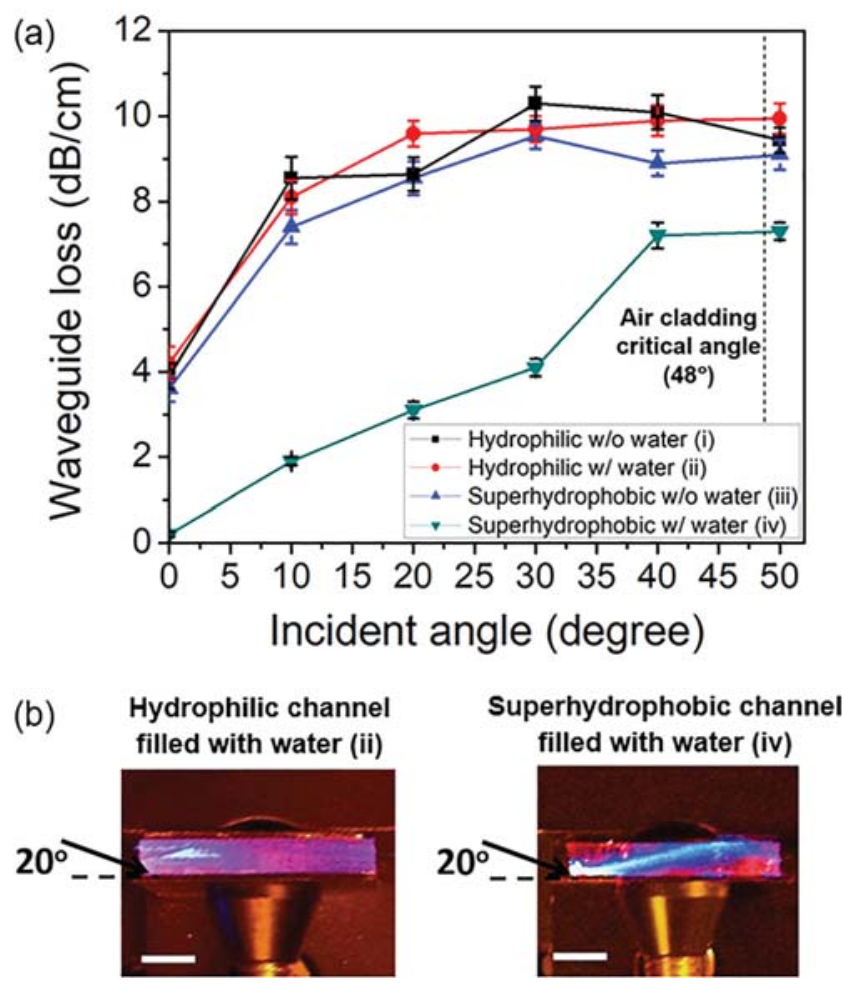

FIG. 3. (a) The measured values of the waveguide losses for the hydrophilic channel without water filled (black, i), the hydrophilic channel with water filled (red, ii), the superhydrophobic channel without water filled (blue, iii), and the superhydrophobic channel with water filled (green, iv). (b) Photographs of the UV lights transmitted along the hydrophilic channel with water filled (ii) and the superhydrophobic channel with water filled (iv) at the incident angle of $20^{\circ}$. The scale bar in each photograph represents $2 \mathrm{~mm}$.

superhydrophobic channels are not significantly different for the various incident angles of the beam, although that of the superhydrophobic channel was slightly (less than $1 \mathrm{~dB} / \mathrm{cm}$ ) smaller in general. While the waveguide loss was $\sim 4 \mathrm{~dB} / \mathrm{cm}$ at the incident angle of $0^{\circ}$, it increased with the incident angle up to $9-10 \mathrm{~dB} / \mathrm{cm}$ until $\sim 20^{\circ}$ and then became almost constant. The large and similar increase in the waveguide loss with respect to the incident angle is mainly due to the broadband absorption of the light by the high-aspect-ratio conical geometry of the sharp-tip nanostructures. ${ }^{31,32}$ With water filled, the waveguide loss for the hydrophilic channel was not significantly different from that without water filled. The refractive index of silicon (3.18) is higher than that of water, so there is effectively no total internal reflection present. Similar to the channels with no water filled, the light is mainly trapped and absorbed by the high-aspect-ratio conical nanostructures with the increase in the incident angle. In contrast, the waveguide loss for the superhydrophobic channel with water filled was significantly lower than the other cases. It was only $\sim 0.1 \mathrm{~dB} /$ $\mathrm{cm}$ at the incident angle of $0^{\circ}$ and not more than $\sim 3 \mathrm{~dB} / \mathrm{cm}$ even at $20^{\circ}$. Unlike the other cases, the waveguide loss kept increasing with the incident angle up to $40^{\circ}-50^{\circ}$, which is around the value of the theoretical critical angle $\left(\sim 48^{\circ}\right)$ for the total internal reflection based on Snell's law ${ }^{33}$ using the effective refractive index of the cladding layer as 1.0. Even with the incident angle around the critical angle, the waveguide loss through the superhydrophobic channel with the liquid core $(\sim 7 \mathrm{~dB} / \mathrm{cm})$ was still lower than the other cases. 
In specific, compared to the hydrophilic channel with water filled, the transmitted power through the superhydrophobic channel with water filled is much higher, such as 83 vs. $10 \%$ and 16 vs. $2 \%$ at the incident angles of 0 and $20^{\circ}$, respectively. As shown in Fig. 3(b), for the case of the incident angle of $20^{\circ}$, the waveguide coupling efficiency of the superhydrophobic channel with the liquid core is evident compared to that of the hydrophilic channel. It indicates that the superhydrophobic channel is indeed effective to create the air cladding layer between the liquid core and solid channel walls to allow the efficient coupling for the total internal reflection and waveguiding. Meanwhile, the superhydrophobic channel with the liquid core still shows the significant waveguide loss even before the incident angle approaches the critical angle (e.g., $\sim 3 \mathrm{~dB} / \mathrm{cm}$ at $20^{\circ}$ ). This is partly due to the increasing effects of the scattering and diffraction of the UV light through the PDMS channel walls (i.e., air/ PDMS/water interfaces at the front and water/PDMS/air interfaces at the end) with a higher incident angle. ${ }^{34}$ Considering the refractive index of PDMS (1.4), the light would also experience the total internal reflection at the PDMS/water interface in the front and the PDMS/air interface at the end with a higher incident angle. In addition, only the top and bottom surfaces of the channels were engineered to be superhydrophobic but not for the sidewalls. Thus, the loss at the sidewalls should also contribute to the waveguide loss with the increase in the incident angle. Furthermore, the scattering by the nanostructures' tips which wet the core water should be more significant as the incident angle increases. Although the PTFE layer for the superhydrophobic channel has a slightly less refractive index than that of water, it was too thin to be effective. Moreover, the effective incident angle to the PTFE layer put on the almost vertical sidewalls of the high-aspect-ratio conical nanostructures is basically beyond the critical angle for the total internal reflection $\left(\sim 11^{\circ}\right)$ so that the PTFE layer should not be effective to suppress the scattering by the solid nanostructures' tips. If such issues are minimized, the superhydrophobic channel with the liquid-core and air-cladding will show more significant waveguide efficacy with much reduced loss.

The results verify that a superhydrophobic channel with a low solid fraction can effectively allow the configuration of a liquid-core air-cladding waveguide platform. Such a unique configuration will be of great significance in optofluidics, where the light can be effectively guided and transmitted along the flow channel with little interference with the solid channel walls. The superhydrophobic waveguide platform also has great potentials to the applications with less friction, Joule heating, and biofouling ${ }^{35}$ taking advantages of the multifunctional benefits of superhydrophobic surfaces. The superhydrophobic waveguide platform can also serve as an additional platform for biosensing based on surfaceenhanced Raman scattering. ${ }^{36}$

This work was supported by the National Science Foundation, Division of Civil, Mechanical and Manufacturing Innovation, under Award No. 1462499.

${ }^{1}$ K. S. Paulsen, D. Di Carlo, and A. J. Chung, "Optofluidic fabrication for 3D-shaped particles," Nat. Commun. 6, 6976 (2015).
${ }^{2}$ J. Sadeghi, A. H. B. Ghasemi, and H. Latifi, "A label-free infrared optofluidic method for real-time determination of flow rate and concentration with temperature cross-sensitivity compensation," Lab Chip 16(20), 3957-3968 (2016).

${ }^{3}$ J. W. Jeong, J. G. McCall, G. Shin, Y. H. Zhang, R. Al-Hasani, M. Kim, S. Li, J. Y. Sim, K. I. Jang, Y. Shi, D. Y. Hong, Y. H. Liu, G. P. Schmitz, L. Xia, Z. B. He, P. Gamble, W. Z. Ray, Y. G. Huang, M. R. Bruchas, and J. A. Rogers, "Wireless optofluidic systems for programmable in vivo pharmacology and optogenetics," Cell 162(3), 662-674 (2015).

${ }^{4}$ K. Du, M. Park, A. Griffiths, R. Carrion, J. Patterson, H. Schmidt, and R. Mathies, "Microfluidic system for detection of viral RNA in blood using a barcode fluorescence reporter and a photocleavable capture probe," Anal. Chem. 89(22), 12433-12440 (2017).

${ }^{5}$ K. Du, H. Cai, M. Park, T. Wall, M. Stott, K. Alfson, A. Griffiths, R. Carrion, J. Patterson, and A. Hawkins, "Multiplexed efficient on-chip sample preparation and sensitive amplification-free detection of Ebola virus," Biosens. Bioelectron. 91, 489-496 (2017).

${ }^{6}$ D. Ozcelik, J. W. Parks, T. A. Wall, M. A. Stott, H. Cai, J. W. Parks, A. R. Hawkins, and H. Schmidt, "Optofluidic wavelength division multiplexing for single-virus detection," Proc. Natl. Acad. Sci. U. S. A. 112(42), 12933-12937 (2015).

${ }^{7}$ J. Sadeghi, D. E. W. Patabadige, A. H. Culbertson, H. Latifi, and C. T. Culbertson, "Out-of-plane integration of a multimode optical fiber for single particle/cell detection at multiple points on a microfluidic device with applications to particle/cell counting, velocimetry, size discrimination and the analysis of single cell lysate injections," Lab Chip 17(1), 145-155 (2017).

${ }^{8}$ F. Tian, J. Min, J. Kanka, X. Li, P. T. Hammond, and H. Du, "Lab-on-fiber optofluidic platform for in situ monitoring of drug release from therapeutic eluting polyelectrolyte multilayers," Opt. Express 23(15), 20132-20142 (2015).

${ }^{9}$ K. Yamada, K. J. Lee, Y. H. Ko, J. Inoue, K. Kintaka, S. Ura, and R. Magnusson, "Flat-top narrowband filters enabled by guided-mode resonance in two-level waveguides," Opt. Lett. 42(20), 4127-4130 (2017).

${ }^{10} \mathrm{~S}$. H. Cho, J. Godin, and Y.-H. Lo, "Optofluidic waveguides in Teflon AFcoated PDMS microfluidic channels," IEEE Photonics Technol. Lett. 21(15), 1057-1059 (2009).

${ }^{11}$ Y. Han, S. Tan, M. K. K. Oo, D. Pristinski, S. Sukhishvili, and H. Du, "Towards full-length accumulative surface — enhanced Raman scattering-active photonic crystal fibers," Adv. Mater. 22(24), 2647-2651 (2010).

${ }^{12} \mathrm{~K}$. Horiuchi and P. Dutta, "Joule heating effects in electroosmotically driven microchannel flows," Int. journal heat mass transfer 47(14-16), 3085-3095 (2004).

${ }^{13} \mathrm{X}$. Peng and G. Peterson, "Convective heat transfer and flow friction for water flow in microchannel structures," Int. journal heat mass transfer 39(12), 2599-2608 (1996).

${ }^{14} \mathrm{H}$. Schmidt and A. R. Hawkins, "Single-virus analysis through chip-based optical detection," Bioanalysis 8(9), 867-870 (2016).

${ }^{15} \mathrm{H}$. Schmidt and A. R. Hawkins, "The photonic integration of non-solid media using optofluidics," Nat. Photonics 5(10), 598-604 (2011).

${ }^{16}$ D. Ozcelik, A. Jain, A. Stambaugh, M. A. Stott, J. W. Parks, A. Hawkins, and H. Schmidt, "Scalable Spatial-Spectral Multiplexing of Single-Virus Detection Using Multimode Interference Waveguides," Sci. Rep. 7(1), 12199 (2017).

${ }^{17}$ D. B. Wolfe, R. S. Conroy, P. Garstecki, B. T. Mayers, M. A. Fischbach, K. E. Paul, M. Prentiss, and G. M. Whitesides, "Dynamic control of liquid-core/liquid-cladding optical waveguides," Proc. Nat. Acad. Sci. United States America 101(34), 12434-12438 (2004).

${ }^{18} \mathrm{G}$. M. Whitesides, "The origins and the future of microfluidics," Nature 442(7101), 368 (2006).

${ }^{19}$ C.-H. Choi and C.-J. Kim, "Large slip of aqueous liquid flow over a nanoengineered superhydrophobic surface," Phys. Rev. Lett. 96(6), 066001 (2006).

${ }^{20}$ C.-H. Choi, U. Ulmanella, J. Kim, C.-M. Ho, and C.-J. Kim, "Effective slip and friction reduction in nanograted superhydrophobic microchannels," Phys. Fluids 18(8), 087105 (2006).

${ }^{21}$ A. Lafuma and D. Quéré, "Superhydrophobic states," Nat. Mater. 2(7), 457 (2003)

${ }^{22}$ M. M. Braun and L. Pilon, "Effective optical properties of non-absorbing nanoporous thin films," Thin Solid Films 496(2), 505-514 (2006).

${ }^{23}$ L. Tong, R. R. Gattass, J. B. Ashcom, S. He, J. Lou, M. Shen, I. Maxwell, and E. Mazur, "Subwavelength-diameter silica wires for low-loss optical wave guiding," Nature 426(6968), 816 (2003).

${ }^{24}$ C. Lee, C.-H. Choi, and C.-J. Kim, "Superhydrophobic drag reduction in laminar flows: A critical review," Experiments Fluids 57(12), 176 (2016). 
${ }^{25}$ H. Jansen, M. de Boer, R. Legtenberg, and M. Elwenspoek, "The black silicon method: A universal method for determining the parameter setting of a fluorine-based reactive ion etcher in deep silicon trench etching with profile control," J. Micromech. Microeng. 5(2), 115 (1995).

${ }^{26}$ S. Koynov, M. S. Brandt, and M. Stutzmann, "Black nonreflecting silicon surfaces for solar cells," Appl. Phys. Lett. 88(20), 203107 (2006).

${ }^{27}$ L. Tsakalakos, J. Balch, J. Fronheiser, M. Y. Shih, S. F. LeBoeuf, M. Pietrzykowski, P. J. Codella, B. A. Korevaar, O. Sulima, J. Rand, A. Davuluru, and U. Rapol, "Strong broadband optical absorption in silicon nanowire films," J. Nanophotonics 1, 013552 (2007).

${ }^{28}$ R. N. Wenzel, "Resistance of Solid Surfaces to Wetting by Water," Ind. Eng. Chem. 28(8), 988-994 (1936).

${ }^{29}$ A. Cassie and S. Baxter, "Wettability of porous surfaces," Tran. Fraday. Soc. 40, 546-551 (1944).

${ }^{30}$ A. Ghatak and K. Thyagarajan, In Optical Waveguides and Fibers, Fundamentals of Photonics, Module 1.7, University of Connecticut, 2000; pp 249-292.
${ }^{31}$ Z. Wang, R. Zhang, S. Wang, M. Lu, X. Chen, Y. Zheng, L. Chen, Z. Ye, C. Wang, and K. Ho, "Broadband optical absorption by tunable Mie resonances in silicon nanocone arrays," Sci. Rep. 5, 7810 (2015).

${ }^{32}$ A. Rahman, A. Ashraf, H. Xin, X. Tong, P. Sutter, M. D. Eisaman, and C. T. Black, "Sub-50-nm self-assembled nanotextures for enhanced broadband antireflection in silicon solar cells," Nat. Comm. 6, 5963 (2015).

${ }^{33}$ J. W. Shirley, "An early experimental determination of Snell's law," Am. J. Phys. 19(9), 507-508 (1951)

${ }^{34}$ G. M. Whitesides and S. K. Tang, In Fluidic Optics, Optofluidics, International Society for Optics and Photonics: 2006; p 63290A.

${ }^{35}$ F. Hizal, N. Rungraeng, J. Lee, S. Jun, H. J. Busscher, H. C. Van der Mei, and C.-H. Choi, "Nanoengineered Superhydrophobic Surfaces of Aluminum with Extremely Low Bacterial Adhesivity," ACS Appl. Mater. Interfaces 9(13), 12118-12129 (2017).

${ }^{36}$ W. Z. Song, D. Psaltis, and K. B. Crozier, "Superhydrophobic bull's-eye for surface-enhanced Raman scattering," Lab a Chip 14(20), 3907-3911 (2014). 